\title{
Spatiotemporal evolution of urban carbon emission performance in China and prediction of future trends
}

\author{
WANG Shaojian ${ }^{1}$, GAO Shuang ${ }^{1}$, HUANG Yongyuan ${ }^{2}$, SHI Chenyi ${ }^{1}$
}

1. Guangdong Provincial Key Laboratory of Urbanization and Geo-simulation, School of Geography and Planning, Sun Yat-sen University, Guangzhou 510275, China;

2. College of Urban and Environmental Sciences, Peking University, Beijing 100871, China

\begin{abstract}
Climate change resulting from $\mathrm{CO}_{2}$ emissions has become an important global environmental issue in recent years. Improving carbon emission performance is one way to reduce carbon emissions. Although carbon emission performance has been discussed at the national and industrial levels, city-level studies are lacking due to the limited availability of statistics on energy consumption. In this study, based on city-level remote sensing data on carbon emissions in China from 1992-2013, we used the slacks-based measure of super-efficiency to evaluate urban carbon emission performance. The traditional Markov probability transfer matrix and spatial Markov probability transfer matrix were constructed to explore the spatiotemporal evolution of urban carbon emission performance in China for the first time and predict long-term trends in carbon emission performance. The results show that urban carbon emission performance in China steadily increased during the study period with some fluctuations. However, the overall level of carbon emission performance remains low, indicating great potential for improvements in energy conservation and emission reduction. The spatial pattern of urban carbon emission performance in China can be described as "high in the south and low in the north," and significant differences in carbon emission performance were found between cities. The spatial Markov probabilistic transfer matrix results indicate that the transfer of carbon emission performance in Chinese cities is stable, resulting in a "club convergence" phenomenon. Furthermore, neighborhood backgrounds play an important role in the transfer between carbon emission performance types. Based on the prediction of long-term trends in carbon emission performance, carbon emission performance is expected to improve gradually over time. Therefore, China should continue to strengthen research and development aimed at improving urban carbon emission performance and achieving the national energy conservation and emission reduction goals. Meanwhile, neighboring cities with different neighborhood backgrounds should pursue cooperative economic strategies that balance economic growth, energy conservation, and emission reductions to realize low-carbon construction and sustainable development.
\end{abstract}

Received: 2020-01-06 Accepted: 2020-03-10

Foundation: Fundamental Research Funds for the Central Universities, No.19lgzd09; Guangdong Special Support Program; Pearl River S\&T Nova Program of Guangzhou, No.201806010187

Author: Wang Shaojian (1986-), Associate Professor, specialized in urban geography and regional development. E-mail: 1987wangshaojian@163.com 
Keywords: urban carbon emission performance; super-efficiency SBM model; spatial Markov chain; spatiotemporal patterns; trend prediction; China

\section{Introduction}

Climate change and its impacts have become one of the most serious environmental problems facing the world today (Su et al., 2013). According to the Fourth Global Climate Assessment of the United Nations Intergovernmental Panel on Climate Change, global warming is an undisputed fact, with human activities and the large amounts of greenhouse gas emissions being the main causes of global climate change. As one of the most important greenhouse gases, $\mathrm{CO}_{2}$ is closely related to global warming (Liu et al., 2019; Wang et al., $2019 b$ ). With the continuous advancement of urbanization and industrialization, $\mathrm{CO}_{2}$ emissions continue to increase. Accordingly, environmental issues caused by $\mathrm{CO}_{2}$ have attracted widespread attention from governments and scholars around the world. To achieve sustainable development, countries have proposed low-carbon action plans, such as the low-carbon action plan in the United Kingdom and the low-carbon society action plan in Japan. Since China is the world's largest $\mathrm{CO}_{2}$ emitter, energy conservation and emission reduction are focal points of the national development strategy in China (Deng et al., 2014; Wang et al., 2019c). China signed the Kyoto Protocol in 2005, and China's nation plan includes clear energy saving and emission reduction targets. For example, China's 12th Five-Year Plan (2011-2015) proposed to reduce carbon emissions by $17 \%$ by 2015 compared to 2010 . The 13th Five-Year Plan (2016-2020) strengthened the goal of low-carbon development, with an $18 \%$ reduction in carbon emissions by 2020 compared with 2015 . In addition, as indicated in the China-United States Joint Statement, China plans to reach a peak in total $\mathrm{CO}_{2}$ emissions by 2030 (Song et al., 2018). A series of planning outlines clearly lay out China's low-carbon

development goals. Research shows that cities are the largest source of energy consumption and greenhouse gas emissions (Ishii et al., 2010). Thus, establishing low-carbon cities is critical for China to deal with climate change and develop a low-carbon economy.

Based on the above considerations, low-carbon development and emission reduction have become hot research topics. The estimation methods (Liu et al., 2011; Zhao et al., 2012), influential factors (Xu et al., 2006; Xu et al., 2012; Ping et al., 2013), intensity (Zhao et al., 2010; Li and Zhou, 2012), and performance (Wang et al., 2010) of $\mathrm{CO}_{2}$ emissions have been extensively studied. Carbon emission performance is an important component in assessing environmental performance. Indicators used to assess carbon emission performance can be divided into single-factor and total-factor indicators according to the measurement methodology. Mielnik and Goldemberg (1999) first evaluated carbon emission performance in developing countries based on the carbon index, a single-factor indicator that reflects the carbon emissions per unit of energy consumption. Yamaji et al. (1993) defined the ratio of total $\mathrm{CO}_{2}$ emissions to GDP as $\mathrm{CO}_{2}$ productivity to study the carbon emission level in Japan. Other single-factor indexes have been used by scholars to evaluate carbon emission performance, including $\mathrm{CO}_{2}$ emission intensity (Sun, 2005), per-capita emissions (Stretesky and Lynch, 2009), cumulative emissions per capita from industrialization (Zhang et al., 2008), and energy intensity (Ang, 1999).

The above single-factor indicators of carbon emission performance are mostly based on the ratio of $\mathrm{CO}_{2}$ emissions to economic or energy-related indexes. While these indicators are 
easy to calculate and understand, they have certain limitations. Because anthropogenic $\mathrm{CO}_{2}$ emissions are mainly derived from economic activities, the essential of carbon emission performance is the input-output efficiency of economic activities, which is not only affected by energy, but also is the result of the joint action of capital, labor and other economic inputs. Therefore, when measuring carbon emission performance, it is necessary to consider the effects of other factors in the process of economic production using total-factor indicators (Zha and Tang, 2012). In total-factor methods, economic production, energy consumption, capital investment, and labor force are considered as the inputs that generate economic products [e.g., gross domestic product (GDP)] as the "expected output" and $\mathrm{CO}_{2}$ as the "non-expected output," making the determination of $\mathrm{CO}_{2}$ emission performance more accurate (Wang et al., 2015). For example, Ramanathan (2002) used the data envelopment analysis (DEA) model to build an input-output indicator system that includes variables related to $\mathrm{CO}_{2}$ emissions, energy consumption, and economic activity and compare carbon emission performance among countries. Subsequently, numerous scholars used this model to calculate the carbon emission performance of different industries in various countries. Zhou et al. (2010) used the DEA model and the Malmquist index to measure the carbon emission performance of 18 countries with high carbon emissions and examine the influential factors. Wang et al. (2013) further analyzed the carbon emission performance of various industrial sectors in China using the DEA method and found that the carbon emission performance of light industry was generally higher than that of heavy industry. Meng et al. (2017) used the range-adjusted measure DEA model to estimate the low-carbon economic efficiency in China's industrial sector from 2001 to 2013; they found that low-carbon economic efficiency remained low in most industries but had greatly improved during the study period. The DEA model focuses only on the expected output of economic activities and ignores the non-expected output, which may lead to errors in the results. Therefore, some scholars have developed improved models to measure carbon emission performance, including the directional distance function model (Du et al., 2014), the slacks-based measure (SBM) model (Wang et al., 2011; Choi et al., 2012; Li, 2013; Wang and Du, 2019a), and the super-efficiency SBM model (Shen et al., 2018). In general, existing studies have analyzed carbon emission performance at the national, regional, and industrial scales. In contrast, few studies have considered carbon emission performance at the city scale.

Based on the above analysis, current research on carbon emission performance is limited by two main deficiencies. First, there is a lack of city-level, energy-related statistical data; most studies have focused on national- or regional-level $\mathrm{CO}_{2}$ emission performance rather than city-scale $\mathrm{CO}_{2}$ emission performance. However, since urban areas are the largest source of energy consumption and greenhouse gas emissions (IEA, 2012), city-level carbon emission performance has important theoretical and practical significance for low-carbon city construction and sustainable economic development. Second, existing studies have mainly focused on the measurement, spatial characteristics, and influencing factors of emission performance, whereas few studies have predicted trends in $\mathrm{CO}_{2}$ emission performance. Predictions of future trends in $\mathrm{CO}_{2}$ emission performance can help realize a balance between economic growth and carbon emission reduction. Therefore, in this study, China's 283 cities were taken as the basic research units, and the super-efficiency SBM model was used to calculate the $\mathrm{CO}_{2}$ emission performance of cities from 1992-2013. The spatial and temporal 
evolution characteristics of urban carbon emission performance were also analyzed. Subsequently, the traditional and spatial Markov transfer probability matrix were constructed to empirically analyze the spatial spillover effect of urban carbon emission performance in China, so as to determine whether the urban carbon emission performance will have an impact on the surrounding areas. Then predict the long-term trends in urban carbon emission performance. The results provide a basis for the science-based formulation of low-carbon sustainable development policies.

\section{Data and methods}

\subsection{Data}

The objective of this study was to measure the carbon emission performance of Chinese cities from an input-output perspective. Considering the lack of data for some cities, the final research objects were 283 cities in China, and the study period was 1992 to 2013. The most important input factors in carbon emission performance are capital, labor, and energy (Zha and Tang, 2012; Wang et al., 2019a). The mechanisms by which these factors affect carbon emissions during economic activities are shown in Figure 1. Based on past research results, fixed-asset investment, the number of employees at year end, and urban electricity consumption were selected as the capital, labor, and energy inputs to the model, respectively. Meanwhile, GDP and urban $\mathrm{CO}_{2}$ emissions were selected as the expected and non-expected outputs during economic activities, respectively. The resulting system of input-output indicators for carbon emission performance in China is depicted in Table 1. Due to the top-down model of energy statistics in China, city-scale energy consumption data are lacking, making it difficult to account for urban carbon emissions. The carbon emissions data in this study were obtained from Wang and Liu (2017) and are based on the simulated inversion of night light data which comes from the Defense Meteorological Satellite Program (DMSP). To ensure the accuracy of these carbon emission data, we compared the simulated carbon emission data derived from Wang and Liu (2017) with the statistically calculated values from some cities with available energy consumption data. The relative error between the simulated and statistically calculated data was $7.65 \%$ (Wang et al., 2018), indicating the good accuracy of the simulated data. Thus, the simulated data were determined to be appropriate

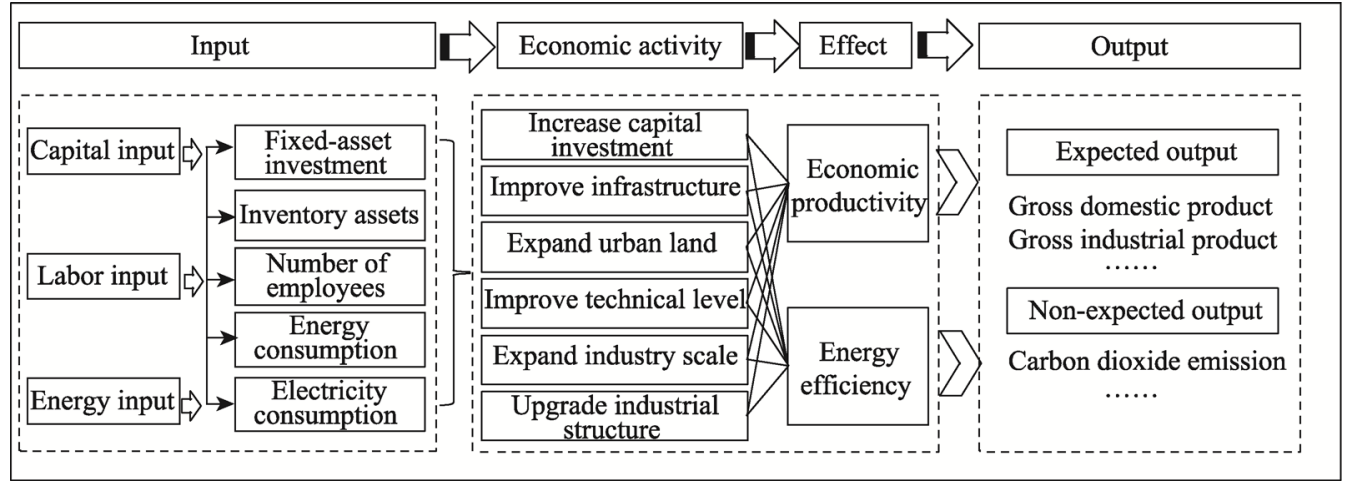

Figure 1 Effects of different factors on urban carbon emission performance from an input-output perspective 
Table 1 System of input-output indicators for carbon emission performance

\begin{tabular}{lllrrrr}
\hline \multicolumn{1}{c}{ Indicator } & \multicolumn{1}{c}{ Variable } & \multicolumn{1}{c}{ Unit } & Mean & Min & Max & S.D. \\
\hline \multirow{3}{*}{ Input } & Fixed-asset investment & $10^{8}$ yuan & 42.65 & 12.95 & 836.24 & 66.34 \\
& Number of employees & $10^{4}$ person & 220.36 & 0.32 & 1729.55 & 169.70 \\
\multirow{2}{*}{ Expected output } & Electricity consumption & $10^{4} \mathrm{kwh}$ & 680.87 & 0.25 & 8514.69 & 907.31 \\
Non-expected output & $\mathrm{CO}_{2}$ emissions & $10^{8}$ yuan & 103.97 & 2.96 & 1483.55 & 125.46 \\
\hline
\end{tabular}

to evaluate urban carbon emission performance in this study. The remaining data were obtained from the China City Statistical Yearbook and related city statistics. Missing data in some years were obtained by linear interpolation. To eliminate the effects of inflation, GDP and fixed-asset investment were converted to 2000 prices.

\subsection{Methods}

\subsubsection{Super-efficiency SBM model based on non-expected output}

In the process of economic production, the input of labor, capital, and energy produces industrial products along with the byproduct $\mathrm{CO}_{2}$, which is regarded as non-expected output. The SBM model originally proposed by Tone (2001) is widely used to study carbon emission performance (Wang and Du, 2019b), ecological efficiency (Zhou et al., 2018), and energy efficiency (Wang et al., 2019) because it accounts for non-expected outputs in the production process, allowing it to accurately reflect actual conditions. Compared to the traditional DEA model, the SBM model considering non-expected outputs has two advantages. First, it can solve the problem of slackness in the calculation of input and output. Second, it can solve the problem of efficiency analysis in the presence of non-expected output (Liu and $\mathrm{Li}, 2015)$. Therefore, in this study, the SBM model based on non-expected output was used to determine $\mathrm{CO}_{2}$ emission performance in Chinese cities.

Suppose a production system with $n$ decision units. Each decision unit is composed of three input-output vectors: input, expected output, and non-expected output. Every $m$ unit of input produces the expected output $S_{1}$ and non-expected output $S_{2}$ (Zhang et al., 2017). The three input-output vectors can be expressed as $x \in R^{m}, y^{g} \in R^{S_{1}}$, and $y^{b} \in R^{S_{2}}$. The matrices $X, Y^{g}$, and $Y^{b}$ are respectively defined as $X=\left[x_{1}, x_{2}, \ldots, x_{n}\right] \in R^{m \times n}, Y^{g}=\left[y_{1}^{g}, y_{2}^{g}, \ldots, y_{n}^{g}\right]$ $\in R^{S_{1} \times n}$, and $Y^{b}=\left[y_{1}^{b}, y_{2}^{b}, \ldots, y_{n}^{b}\right] \in R^{S_{2} \times n}$.

Assuming $X>0, Y^{g}>0$, and $Y^{b}>0$, the production possibility set can be defined as (Zhang et al., 2017)

$$
\mathrm{P}=\left\{\left(x, y^{g}, y^{b}\right) \mid x \geqslant X \theta, y^{g} \geqslant Y^{g} \theta, y^{b} \leqslant Y^{b} \theta, \theta \geqslant 0\right\}
$$

The actual expected output level is lower than the ideal expected output level, and the actual non-expected output level is higher than the ideal non-desired output level (Hong et al., 2013). In Tone's SBM model, non-expected outputs are incorporated into the evaluation decision unit $\left(x_{0}, y_{0}^{g}, y_{0}^{b}\right)$ as follows (Tone, 2001):

$$
\rho=\min \frac{1-\frac{1}{m} \sum_{i=1}^{m} \frac{S_{i}^{-}}{x_{i 0}}}{1+\frac{1}{S_{1}+S_{2}}\left(\sum_{r=1}^{S_{1}} \frac{S_{r}^{g}}{y_{r 0}^{g}}+\sum_{r=1}^{S_{2}} \frac{S_{r}^{b}}{y_{r 0}^{b}}\right)}
$$




$$
\text { s.t. }\left\{\begin{array}{c}
x_{0}=X \theta+S^{-} \\
y_{0}^{g}=Y^{g} \theta-S^{g} \\
y_{0}^{b}=Y^{b} \theta-S^{b} \\
S^{-} \geqslant 0, S^{g} \geqslant 0, S^{b} \geqslant 0, \theta \geqslant 0
\end{array}\right.
$$

where $S=\left(S^{-}, S^{g}, S^{b}\right)$ is the amount of slack in the input, expected output, and non-expected output; and $\rho$ is the efficiency of the decision unit, which ranges from 0 to 1 . For a given decision unit $\left(x_{0}, y_{0}^{g}, y_{0}^{b}\right)$, the decision unit is valid if and only if $\rho=1$; that is, $S^{-}=S^{g}=S^{b}=0$. Through the Charnes-Cooper transformation, the above nonlinear equation is transformed into a linear model, which is more conducive to calculation. The equivalent form of the model is (Tone, 2001)

$$
\text { s.t. }\left\{\begin{array}{c}
\tau=\operatorname{mint}-\frac{1}{m} \sum_{i=1}^{m} \frac{S_{i}^{-}}{x_{i 0}} \\
1=t+\frac{1}{S_{1}+S_{2}}\left(\sum_{r=1}^{S_{1}} \frac{S_{r}^{g}}{y_{r 0}^{g}}+\sum_{r=1}^{S_{2}} \frac{S_{r}^{b}}{y_{r 0}^{b}}\right) \\
x_{0} t=X \mu+S^{-} \\
y_{0}^{g} t=Y^{g} \mu-S^{g} \\
y_{0}^{b} t=Y^{b} \mu-S^{b} \\
S^{-} \geqslant 0, S^{g} \geqslant 0, S^{b} \geqslant 0, \mu \geqslant 0, t>0
\end{array}\right.
$$

In most evaluations of efficiency, multiple decision-making units have $100 \%$ efficiency values. Therefore, when ranking these efficiencies, it is important to distinguish between these efficiency decision-making units. Considering the findings of Tone (2002), we chose the super-efficiency SBM model, which is expressed as follows:

$$
\begin{gathered}
\rho^{*}=\min \frac{\frac{1}{m} \sum_{i=1}^{m} \frac{\bar{x}_{i}}{x_{i 0}}}{\frac{1}{S_{1}+S_{2}}\left(\sum_{r=1}^{S_{1}} \frac{\bar{y}_{r}^{g}}{y_{r 0}^{g}}+\sum_{r=1}^{S_{2}} \frac{\bar{y}_{r}^{b}}{y_{r 0}^{b}}\right)} \\
\text { s.t. }\left\{\sum_{j=1, \neq k}^{n} \theta_{j} x_{j}\right. \\
\bar{y}^{g} \leqslant \sum_{j=1, \neq k}^{n} \theta_{j} y_{j}^{g} \\
\bar{y}^{b} \geqslant \sum_{j=1, \neq k}^{n} \theta_{j} y_{j}^{b} \\
\bar{x} \geqslant x_{0}, \bar{y}^{g} \leqslant y_{0}^{g}, \bar{y}^{b} \geqslant y_{0}^{b}, \bar{y}^{g} \geqslant 0, \theta \geqslant 0
\end{gathered}
$$

where $\rho^{*}$ is the efficiency value of the decision unit, and its value can exceed 1 . The above 
models assume that the scale is constant.

\subsubsection{Spatial Markov chain}

Revealing the spatiotemporal dynamics of an area is an important part of geographic research. Coefficients such as the Theil index, Syr coefficient, and coefficient of variation can be combined with exploratory spatial statistical methods to reflect the temporal evolution and spatial distribution characteristics of geographic phenomena. However, the above methods rely on static processes to reflect regional phenomena and ignore the dynamic characteristics of the region. Meanwhile, the above methods are best suited to provide information for the entire region; they cannot reflect internal dynamic information. To address the shortcomings of the above methods, the Markov chain method treats the evolution of regional phenomena as a Markov process to reflect the static and dynamic characteristics in each region (Le, 2004; Wei and Liao, 2012).

The Markov chain is based on continuous values quantified by regional phenomena and discretizes regional phenomena into $k$ state types. By calculating the various types of changes and their probabilities, the evolution of regional phenomena can be approximated as a Markov process. First, a $1 \times k$ matrix $F_{t}=\left[F_{1, t}, F_{2, t}, \cdots, F_{k, t}\right]$ is used to store the state probability of carbon emission performance in each region in period $t$. The transfer of regional carbon emission performance in different periods is shown in Table 2 , which is a $k \times k$ Markov transfer probability matrix $M$. Herein, a city's carbon emission performance value is divided into four types according to the quartile $(0.25,0.5,0.75)$, and the types are $k=1,2$, 3,4 . The element $P_{i j}$ in the matrix in Table 2 represents the probability that the city's carbon emission performance value is type $i$ at time $t$ and type $j$ at time $t+1 . P_{i j}$ is calculated as

$$
P_{i j}=\frac{z_{i j}}{z_{i}},
$$

where $z_{i j}$ represents the sum of the number of study areas that transfer from type $i$ at time $t$ to type $j$ at time $t+1$ during the entire study period, and $z_{i}$ is the sum of the number of regions belonging to type $i$ during the entire study period.

Table 2 Markov transfer probability matrix $(k=4)$

\begin{tabular}{ccccc}
\hline$t / t+1$ & 1 & 2 & 3 & 4 \\
\hline 1 & $P_{11}$ & $P_{12}$ & $P_{13}$ & $P_{14}$ \\
2 & $P_{21}$ & $P_{22}$ & $P_{23}$ & $P_{24}$ \\
3 & $P_{31}$ & $P_{32}$ & $P_{33}$ & $P_{34}$ \\
4 & $P_{41}$ & $P_{42}$ & $P_{43}$ & $P_{44}$ \\
\hline
\end{tabular}

When studying different periods, scholars have found that spatial spillover due to geographical proximity plays an important role in regional development and evolution (Rey, 2010). Considering the spatial characteristics of regional phenomena, we introduced the concept of spatial lag to decompose the traditional Markov chain $k \times k$ transfer probability matrix into $k k \times k$ transfer condition probability matrices. As shown in Table 3, the element $P_{(l, i, j)}$ in the matrix represents the probability that a city's carbon emission performance value transfers from the initial state type $i$ to type $j$ at the next time point when the spatial lag type is $l$. The spatial lag type considers the units that are geographically adjacent to the area. The specific formula is as follows:

$$
\operatorname{Lag}_{a}=\sum_{b=1}^{n} Y_{b} W_{a b}
$$


where $Y_{b}$ is the observed value of area $b, \operatorname{Lag}_{a}$ is the spatial lag of area $a, n$ is the total number of cities, and the spatial weight matrix $W_{a b}$ reflects the spatial relationship between areas $a$ and $b$. In this study, the adjacency principle was adopted to define the spatial relationship; that is, the value is 1 for the adjacent region and 0 otherwise. Due to the island problem caused by the lack of statistical data, we define the city closest to the island as the adjacent city.

Table 3 Spatial Markov transfer probability matrix $(k=4)$

\begin{tabular}{|c|c|c|c|c|c|}
\hline Lag & $t / t+1$ & 1 & 2 & 3 & 4 \\
\hline \multirow{4}{*}{1} & 1 & $P_{11 \mid 1}$ & $P_{12 \mid 1}$ & $P_{13 \mid 1}$ & $P_{14 \mid 1}$ \\
\hline & 2 & $P_{21 \mid 1}$ & $P_{22 \mid 1}$ & $P_{23 \mid 1}$ & $P_{24 \mid 1}$ \\
\hline & 3 & $P_{31 \mid 1}$ & $P_{32 \mid 1}$ & $P_{33 \mid 1}$ & $P_{34 \mid 1}$ \\
\hline & 4 & $P_{41 \mid 1}$ & $P_{42 \mid 1}$ & $P_{43 \mid 1}$ & $P_{44 \mid 1}$ \\
\hline \multirow{4}{*}{2} & 1 & $P_{11 \mid 2}$ & $P_{12 \mid 2}$ & $P_{13 \mid 2}$ & $P_{14 \mid 2}$ \\
\hline & 2 & $P_{21 \mid 2}$ & $P_{22 \mid 2}$ & $P_{23 \mid 2}$ & $P_{24 \mid 2}$ \\
\hline & 3 & $P_{31 \mid 2}$ & $P_{32 \mid 2}$ & $P_{33 \mid 2}$ & $P_{34 \mid 2}$ \\
\hline & 4 & $P_{41 \mid 2}$ & $P_{42 \mid 2}$ & $P_{43 \mid 2}$ & $P_{44 \mid 2}$ \\
\hline \multirow{4}{*}{3} & 1 & $P_{11 \mid 3}$ & $P_{12 \mid \beta}$ & $P_{13 \mid 3}$ & $P_{14 \mid 3}$ \\
\hline & 2 & $P_{21 \mid 3}$ & $P_{22 \mid \beta}$ & $P_{23 \mid 3}$ & $P_{24 \mid 3}$ \\
\hline & 3 & $P_{31 \mid 3}$ & $P_{32 \mid 3}$ & $P_{33 \mid 3}$ & $P_{34 \mid 3}$ \\
\hline & 4 & $P_{41 \mid 3}$ & $P_{42 \mid 3}$ & $P_{43 \mid 3}$ & $P_{44 \mid 3}$ \\
\hline \multirow{4}{*}{4} & 1 & $P_{11 \mid 4}$ & $P_{12 \mid 4}$ & $P_{13 / 4}$ & $P_{144 \mid}$ \\
\hline & 2 & $P_{21 \mid 4}$ & $P_{2214}$ & $P_{23 \mid 4}$ & $P_{2444}$ \\
\hline & 3 & $P_{31 \mid 4}$ & $P_{32 \mid 4}$ & $P_{33 \mid 4}$ & $P_{34 \mid 4}$ \\
\hline & 4 & $P_{41 \mid 4}$ & $P_{42 \mid 4}$ & $P_{43 \mid 4}$ & $P_{44 \mid 4}$ \\
\hline
\end{tabular}

After a long period of transfer, the system will reach a stable state that is not affected by time. The corresponding probability at this moment is called the ultimate state probability or the equilibrium state probability. By referring to the definition of limit, the Markov transfer probability matrix can be used to calculate the ultimate state. To some extent, this ultimate state probability provides important information for predicting the future trend in the Markov process. The calculation formula is as follows:

$$
\lim _{k \rightarrow \infty} \pi(k)=\lim _{k \rightarrow \infty} \pi(k+1)=\pi
$$

where $\pi$ is the ultimate state matrix of the evolution of the Markov process. If $\pi$ satisfies $\sum_{i=1}^{n} \pi_{i}=1$ and $0 \leqslant \pi_{i} \leqslant 1, \pi$ is the ultimate state of the traditional Markov process. Substituting Eq. (10) into the recursive formula of the Markov prediction model gives

$$
\lim _{k \rightarrow \infty} \pi(k+1)=\lim _{k \rightarrow \infty} \pi(k) M
$$

Similarly, the method for calculating the final state of a traditional Markov process can be extended to a spatial Markov chain to calculate the final state of the Markov process under different spatial lag states. This provides important information about the evolution of the spatial Markov process. 


\section{Spatiotemporal evolution of carbon emission performance in Chinese cities}

\subsection{Measurement and spatiotemporal distribution of carbon emission performance}

The super-efficiency SBM model was used to determine the carbon emission performance of 283 cities in China from 1992 to 2013 and analyze the spatiotemporal evolution in carbon emission performance. Figure 2 shows the average change in carbon emission performance across China, while Figure 3 shows a box plot of carbon emission performance in Chinese cities. As shown in Figure 2, the overall level of carbon emission performance in Chinese cities remains

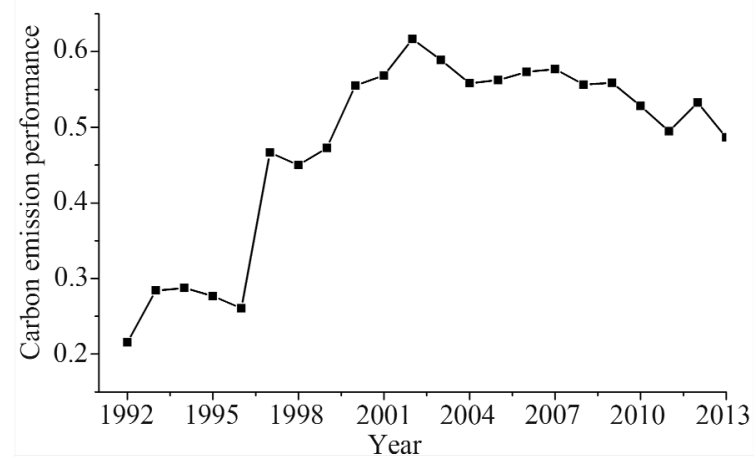

Figure 2 Evolution in urban carbon emission performance from 1992-2013 low $(<0.6)$. This indicates the existence of significant room for improvement in China's economic development in terms of technological progress and emission reduction. Because the Chinese government prioritizes low-carbon city construction, energy conservation, and emission reduction, China adopted a series of measures to improve carbon emission efficiency between 1995 and 2002. However, given the bottlenecks in energy conservation and emission reduction, carbon emission performance in China has stagnated. Thus, a new round of industrial transformation and energy structure adjustment is needed to improve carbon emission performance in China. Figure 3 shows the changes in the distribution of carbon emission performance in Chinese cities from 1992 to 2013. During the study period, the overall carbon emission performance greatly improved. The change in the mean value of carbon emission performance in each city has been concentrated from low value to median value. Many cities with low carbon emissions have significantly improved their carbon emission performance by investing in technology and modernizing their industries. However, the carbon emission performance remains low in some cities. In general, the carbon emission performance of Chinese cities differs signify-

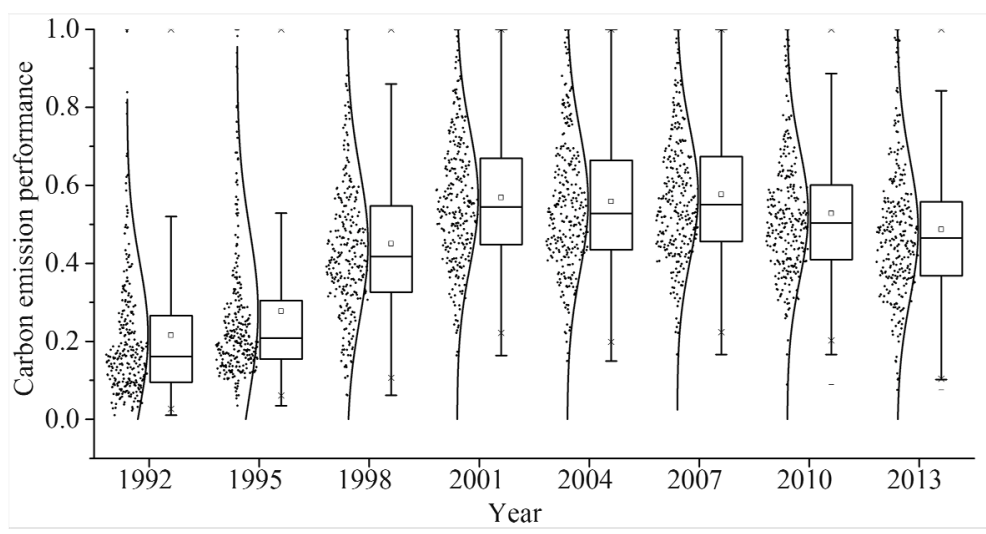

Figure 3 Box plot of urban carbon emission performance in Chinese cities from 1992 to 2013 
cantly.

ArcGIS 10.3 software was used to visualize the carbon emission performance of Chinese cities. Figure 4 shows the spatial distribution of carbon emission performance in Chinese cities. Figure 4 indicates that the carbon emission performance in most cities improved significantly during the study period, especially from 1995 to 2000. Since 2000, cities in Hunan, Hubei, Jiangxi, Zhejiang, Jiangsu, Anhui, Shandong, and Liaoning provinces have greatly improved their carbon emission performance, while smaller improvements in carbon emission performance were observed in cities in Shaanxi, Shanxi, Hebei, Guangxi, and Guangdong provinces. Notably, the carbon emission performance of cities in Heilongjiang and Jilin provinces was relatively high from 2000-2005 and then declined to a certain extent. In addition, the carbon emission performance in cities in Shaanxi, Hebei, Henan, and Guangdong provinces all declined from 2010-2013. In 2013, the spatial pattern of carbon emission performance in Chinese cities could be described as "high in the south and low in the north." In this pattern, carbon emission performance gradually decreased moving outward from Hunan and Sichuan.

(a) 1992

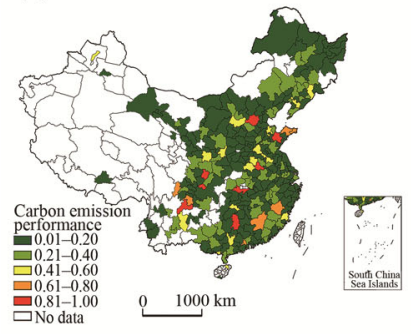

(d) 2005

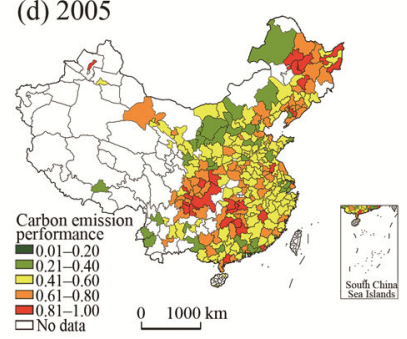

(b) 1995

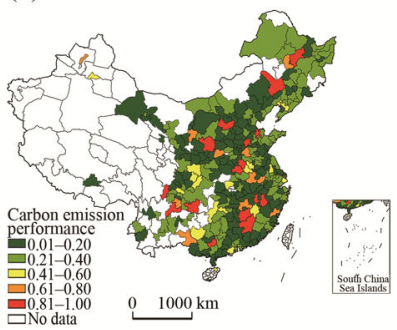

(e) 2010

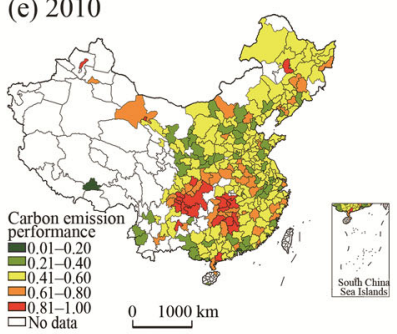

(c) 2000

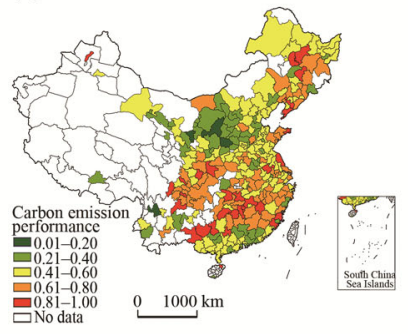

(f) 2013

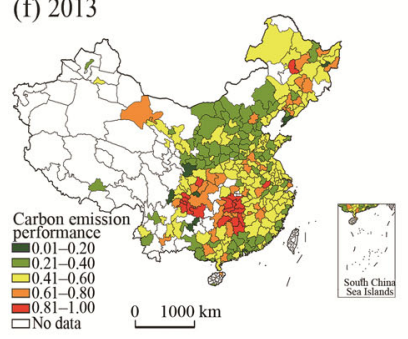

Figure 4 Spatial distributions of urban carbon emission performance in Chinese cities from 1992-2013

\subsection{Spatiotemporal evolution of carbon emission performance}

The traditional Markov transfer and spatial Markov transfer probability matrices were constructed and used to analyze the spatiotemporal evolution of carbon emission performance in Chinese cities. In each matrix, carbon emission performance is discretely divided into four states: low, medium-low, medium-high, and high (respectively represented by $k=1,2,3$, and 4). The transfer from a low value to a high value is defined as a downward transfer, while the transfer from a high value to a low value is defined as an upward transfer. The traditional Markov transfer probability matrix shown in Table 4 indicates the following. (1) The diagonal probabilities are greater than the non-diagonal probabilities, suggesting that 
urban carbon emission performance in China is stable, with a high probability of maintaining the original state. (2) A "club convergence" phenomenon is observed in the carbon emission performance of Chinese cities. That is, different regions will form different clubs in the transfer of carbon emission performance according to their initial conditions, and regions with similar conditions within the club will converge in the transfer. (3) The cities with low and high carbon emission performance have the highest probability of maintaining the original state at the next time period $(74.37 \%$ and $80.13 \%$, respectively). The probability of transfer between urban carbon emission performance types in adjacent years is low $(<6 \%)$.

Table 4 Markov matrix of city-level carbon emission performance types from 1992-2013

\begin{tabular}{cccccc}
\hline$t / t+1$ & $n$ & 1 & 2 & 3 & 4 \\
\hline 1 & 1514 & 0.7437 & 0.1777 & 0.0575 & 0.0211 \\
2 & 1457 & 0.1030 & 0.6603 & 0.1929 & 0.0439 \\
3 & 1472 & 0.0177 & 0.1793 & 0.6372 & 0.1658 \\
4 & 1500 & 0.0133 & 0.0253 & 0.1600 & 0.8013 \\
\hline
\end{tabular}

To construct the spatial Markov transfer probability matrix, a spatial lag condition was added to the traditional Markov chain transfer probability matrix. By comparing the probabilities of transfers in urban carbon emission performance type under different neighborhood backgrounds, the effect of different carbon emission performance levels in surrounding cities on urban carbon emission performance transfer was explored. Based on the spatial Markov transfer probability matrix in Table 5, the following conclusions can be drawn. (1) Neighborhood background plays an important role in the transfer of carbon emission performance in Chinese cities. Compared to the traditional Markov transfer probability matrix, the carbon emission performance transfer probability in Chinese cities has changed significantly under different neighborhood backgrounds. (2) The transfer of urban carbon performance is synergistic with the type of regional carbon emission performance. For example, when the city's neighborhood background is 1 , the number of cities with low carbon emission performance at time $t$ is significantly more than for other types of cities. When the city's neighborhood background is 4 , the number of cities with high carbon emission performance at time $t$ is far more than the number of other types. (3) Generally speaking, when a neighboring city has a low carbon emission performance, the probability of an upward transfer in the city's carbon emission performance increases; conversely, when a neighboring city has a high carbon emission performance, the city's carbon emission performance is more likely to shift downward. For example, as shown in Table 5, in neighborhood conditions with low carbon emission performance, $P_{21 \mid 1}(0.1565)>P_{21}(0.1029), P_{32 \mid 1}(0.2330)>$ $P_{32}$ (0.1793). However, in neighborhood conditions where carbon emission performance is high, $P_{12 \mid 4}(0.3272)>P_{12}(0.1776), P_{23 \mid 4}(0.2089)>P_{23}(0.1928)$. (4) The spatial Markov transfer probability matrix provides an explanation for the club convergence phenomenon in the spatial dimension. Affected by the spillover effect of neighborhood backgrounds, the transfer of carbon emission performance type in Chinese cities forms this club convergence phenomenon within a certain geographic space. That is, in regions with low carbon emission performance, the probability of a downward transfer in carbon emission performance increases, $P_{21 \mid 1}(0.1565)>P_{21 \mid 4}(0.0709), P_{32 \mid 1}(0.2330)>P_{32 \mid 4}(0.01489)$, while the probability 
of an upward transfer decreases, $P_{12 \mid 1}(0.1437)<P_{12 \mid 4}(0.3273), P_{23 \mid 1}(0.1821)<P_{23 \mid 4}(0.2090)$. Therefore, cities with low carbon emission performance will accumulate in this geographic area. Similarly, the number of high-performance cities will increase over time in regions with high carbon emission performance.

Table 5 Spatial Markov matrix of city-level carbon emission performance in China from 1992-2013

\begin{tabular}{|c|c|c|c|c|c|c|}
\hline Lag & $t / t+1$ & $n$ & 1 & 2 & 3 & 4 \\
\hline \multirow{4}{*}{1} & 1 & 807 & 0.7720 & 0.1437 & 0.0595 & 0.0248 \\
\hline & 2 & 313 & 0.1565 & 0.6006 & 0.1821 & 0.0607 \\
\hline & 3 & 206 & 0.0388 & 0.2330 & 0.5631 & 0.1650 \\
\hline & 4 & 176 & 0.0341 & 0.0455 & 0.1364 & 0.7841 \\
\hline \multirow{4}{*}{2} & 1 & 470 & 0.7319 & 0.2000 & 0.0553 & 0.0128 \\
\hline & 2 & 436 & 0.1124 & 0.6651 & 0.1789 & 0.0436 \\
\hline & 3 & 321 & 0.0218 & 0.2274 & 0.5919 & 0.1589 \\
\hline & 4 & 256 & 0.0430 & 0.0313 & 0.1953 & 0.7305 \\
\hline \multirow{4}{*}{3} & 1 & 182 & 0.6923 & 0.2253 & 0.0495 & 0.0330 \\
\hline & 2 & 440 & 0.0750 & 0.6841 & 0.2045 & 0.0364 \\
\hline & 3 & 475 & 0.0147 & 0.1537 & 0.6505 & 0.1811 \\
\hline & 4 & 371 & 0.0054 & 0.0296 & 0.2075 & 0.7574 \\
\hline \multirow{4}{*}{4} & 1 & 55 & 0.6000 & 0.3273 & 0.0727 & 0.0000 \\
\hline & 2 & 268 & 0.0709 & 0.6828 & 0.2090 & 0.0373 \\
\hline & 3 & 470 & 0.0085 & 0.1489 & 0.6872 & 0.1553 \\
\hline & 4 & 697 & 0.0014 & 0.0158 & 0.1277 & 0.8551 \\
\hline
\end{tabular}

Considering the neighborhood background conditions, the transfer of carbon emission performance in Chinese cities has changed significantly over time, indicating that the evolution of urban carbon emission performance exhibits a spatial spillover effect. To determine whether this spatial spillover effect is statistically significant, a hypothesis test was required. The transfer of a city's carbon emission performance was assumed to be independent of each other and independent of neighborhood background. The following formula was used:

$$
Q_{b}=-2 \log \left\{\prod_{l=1}^{k} \prod_{i=1}^{k} \prod_{j=1}^{k}\left[\frac{P_{i j}}{P_{(l, i, j)}}\right]^{n_{(l, i, j)}}\right\}
$$

where $Q_{b}$ is asymptotically distributed as $x^{2}$ with $k(k-1)$ degrees of freedom, $k$ is the the number of types in the distribution: $k=4, P_{i j}$ is the traditional Markov transfer probability, $P_{(l, i, j)}$ is the spatial Markov transfer probability of neighborhood background $l$, and $n_{(l, i, j)}$ is the number of cities with spatial Markov transfers in the neighborhood background $l$. At a confidence level of $\alpha=0.005, Q_{b}=109.15>x^{2}(40)=66.77$; therefore, the assumption that the transfer of carbon emission performance in Chinese cities is spatially independent is not valid.

The spatial Markov transfer probability matrix indicates that the evolution of carbon emission performance in Chinese cities exhibits a significant spatial spillover effect. The 
main manifestation of this spillover effect is the consistency of the city-level carbon emission performance with the regional performance. Meanwhile, the transfer of carbon emission performance in Chinese cities is affected by the spillover effects of neighborhood background, resulting in the club convergence phenomenon within certain geographical spaces. Neighborhood background plays an important role in the transfer of carbon emission performance in Chinese cities. As shown in Figure 1, the regional carbon emission performance results from the combined effect of expected and non-expected outputs and is dominated by regional economic activities. Therefore, the spatial spillover effect in regional carbon emission performance is a concrete manifestation of the spatial spillover effect in regional economic activities. The specific mechanism is as follows.

According to the theory of economic growth, regional economic activities are the result of a combination of factors including capital, markets, technology, and policy. Under specific capital conditions, technological levels, and institutional environments, various economic factors interact to form the model of regional economic development (Zeng et al., 2015). First, the emergence of a unified market has strengthened the free flow of economic factors between cities. Second, an open economy allows cities to learn from advanced technologies in other regions, especially in neighboring areas. In addition, the diminishing marginal utility of policy innovation has promoted intergovernmental institutional learning and imitation. For these reasons, regional economic development tends to be synergetic, resulting in a spatial spillover effect in economic activities. In particular, the development of informatization and the continuous improvement in regional infrastructure have reduced the flow cost of interregional factors, promoted the flow of interregional factors, promoted the free flow of interregional factors, and made the spatial spillover in economic activities more significant (Zhang, 2012). On one hand, the spatial spillover effect in regional economic activities has changed the efficiency of regional economic output; thus, the expected output of regional economic activities has a spatial spillover effect in the development process. On the other hand, due to the strong energy dependence of economic activities in Chinese cities (World, 2010), the coordinated transformation of regional economic development models has led to a coordinated transformation of energy consumption patterns and energy efficiency within regions (Wang and Huang, 2019). Therefore, the non-expected output of economic activities also exhibits a spatial spillover effect. The spatial spillover in economic activity between regions is affected by interactions among multiple factors, which affect regional carbon emission performance by changing the expected and non-expected outputs of economic activities. In general, the spatial spillover effect in carbon emission performance results from the combined action of multiple geographic factors (e.g., markets, technologies, and institutions).

\section{Prediction of trends in carbon emission performance in Chinese cities}

The limit distribution of Markov transfer probability is the distribution in equilibrium state after long time transfer system. The corresponding probability is called the equilibrium state probability, which can effectively predict the long-term evolution in carbon emission performance. When $k \rightarrow \infty$, the limit distribution of the state types in China's urban carbon emission performance after $k$ transfers can be obtained. After adding the spatial lag component, we can analyze the limit distribution of urban carbon emission performance status 
types in each neighborhood background. Table 6 shows the predicted trends in carbon emission performance in Chinese cities. Based on a comparison of the initial state and the limit distribution state of the traditional Markov transfer probability matrix, the number of states 1 , 2 , and 3 is predicted to decrease, while the number of state 4 is predicted to increase significantly. Thus, in the long term, carbon emission performance in Chinese cities will gradually shift from low to high; that is, carbon emission performance will gradually improve. In addition, when accounting for spatial lag, China's urban carbon emission performance still shows a significant upward trend. In the long term, the numbers of states 1, 2, 3, and 4 in cities adjacent to regions with low carbon emission performance (type 1) are similar. However, when adjacent regions have high carbon emission performance (types 3 and 4), the number of cities with high carbon performance will be much higher than the number of cities with low carbon performance.

Generally speaking, the long-term evolution in China's urban carbon emission performance is optimistic. The carbon emission performance is expected to improve gradually over time, with the numbers of cities with low and high carbon emission performance decreasing and increasing, respectively. The effect of neighborhood background on the evolution of urban carbon emission performance is heterogeneous. Cities adjacent to low-performance areas will have a smaller increase in carbon emission performance with a relatively equal distribution among the four state types. In contrast, in cities adjacent to high-performance areas, the carbon emission performance types are clustered around types 3 and 4, and an overall upward trend is predicted.

Table 6 Predicted evolution in carbon emission performance in Chinese cities

\begin{tabular}{|c|c|c|c|c|c|c|}
\hline State & ype & & 1 & 2 & 3 & 4 \\
\hline \multirow{2}{*}{ Ignoring spatial lag } & \multicolumn{2}{|l|}{ Initial state } & 0.1484 & 0.3534 & 0.3004 & 0.1979 \\
\hline & \multicolumn{2}{|l|}{ Ultimate state } & 0.1377 & 0.2512 & 0.2948 & 0.3162 \\
\hline \multirow{4}{*}{ Considering spatial lag } & \multirow{4}{*}{ Ultimate state } & 1 & 0.2521 & 0.2524 & 0.2242 & 0.2713 \\
\hline & & 2 & 0.1908 & 0.3184 & 0.2708 & 0.2201 \\
\hline & & 3 & 0.0851 & 0.2585 & 0.3471 & 0.3093 \\
\hline & & 4 & 0.0477 & 0.2220 & 0.3249 & 0.4054 \\
\hline
\end{tabular}

\section{Discussion and conclusions}

Taking prefecture-level cities in China as the research units and 1992-2013 as the study period, the super-efficiency SBM model was used to evaluate urban carbon emission performance. In addition, the traditional and spatial Markov chain-based transfer probability matrices were constructed to conduct an empirical analysis of the spatial spillover effects of carbon emission performance in Chinese cities. The spatial and temporal evolution characteristics of urban carbon emission performance were then evaluated, and the future trends in urban carbon emission performance were predicted. The main conclusions are summarized as follows.

Considering the spatial and temporal distributions, urban carbon emission performance in China steadily increased during the study period with some fluctuations. However, the overall level of urban carbon emission performance remained low, indicating considerable room 
for improvement. The change in the mean value of carbon emission performance in each city has been concentrated from low value to median value. Prominent differences were observed in carbon emission performance between cities. Spatially, the pattern of carbon emission performance in China could be described as "high in the south and low in the north"; carbon emission performance gradually decreased moving outward from Hunan and Sichuan.

The traditional Markov probability transfer matrix indicates that carbon emission performance in Chinese cities is relatively stable, and a club convergence phenomenon is observed. The diagonal probabilities are greater than the non-diagonal probabilities, suggesting that urban carbon emission performance in China is stable. The spatial Markov transfer probability matrix indicates that neighborhood background plays an important role in the transfer of carbon emission performance in Chinese cities. For example, when a city is adjacent to a region with low carbon emission performance, the probability of an upward transfer in the city's carbon emission performance increases; conversely, when a city is adjacent to a region with high carbon emission performance, the probability of a downward transfer increases. The spatial Markov transfer probability matrix also provides an explanation for the observed club convergence phenomenon in the spatial dimension based on the spatial spillover effects of neighborhood background.

The predicted long-term trends in carbon emission performance in China are optimistic. Carbon emission performance is predicted to gradually shift from low to high over time. The influence of neighborhood background on the evolution of urban carbon emission performance is heterogeneous. Cities adjacent to low-performing areas will have a smaller increase in carbon emission performance than cities adjacent to high-performing areas.

The carbon emission performance in economically developed regions is lower than that of less developed regions in central and western China. This indicates that the economic development in China still conforms to the "high energy consumption, high emissions, and high growth" model (Zha and Tang, 2012). Although the Chinese government has successively introduced a series of policies and measures with promising results, China is still in the rising portion of the "inverted U-shape" of Kuznets curve of economic development and carbon emissions (Wei and Yu, 2011). The trends in carbon emission performance predicted in this study indicate a large gap in performance between cities, and carbon emission performance in some cities can be greatly improved. In the future, China should continue to improve urban carbon emission performance and make progress toward national energy conservation and emission reduction goals (Wang et al., 2016; 2017). The Markov matrix and spatial Markov matrix constructed in this study show that the city-level spatial characteristics of carbon emission performance in China are similar to the regional characteristics. Thus, neighboring cities should coordinate their economic development efforts. In particular, cities adjacent to high-value carbon emission performance areas should strengthen their industrial, technological, and institutional exchanges with high-value areas to improve carbon emission performance. Meanwhile, the government should strengthen cooperation among cities and formulate energy conservation and emission reduction policies from a regional perspective. These measures will help achieve China's goals of establishing low-carbon cities and developing a low-carbon economy (Song et al., 2018).

Finally, this study has some shortcomings. First, the use of night light data to simulate urban carbon emissions may result in some error. Second, the existing DMSP light image 
data are only published for years up to 2013; more recent data are needed to improve the timeliness of this study. In recent years, China's economic development has changed greatly, profoundly affecting carbon emission performance. In future work, these data issues should be resolved to generate more timely results. Finally, this study used alternative data to establish the input-output indicator system. While these data are available and convenient, their accuracy is not sufficient. For example, electricity consumption was used in place of energy input, and the differences in energy consumption between cities were not considered. In addition, the number of employees at year end was used instead of labor input, and the effects of differences in the type and quality of labor among industrial structures were ignored. Future research should further emphasize data comprehensiveness and accuracy.

\section{References}

Ang B W, 1999. Is the energy intensity a less useful indicator than the carbon factor in the study of climate change? Energy Policy, 27(15): 943-946.

Choi Y, Ning Z, Zhou P, 2012. Efficiency and abatement costs of energy-related $\mathrm{CO}_{2}$ emissions in China: A slacks-based efficiency measure. Applied Energy, 98: 198-208.

Deng J X, Liu X, W Z, 2014. Characteristics analysis and factor decomposition based on the regional difference changes in China's $\mathrm{CO}_{2}$ emission. Journal of Natural Resources, 29(2): 189-200. (in Chinese)

$\mathrm{Du} \mathrm{K}$ R, Huang L, Yu K, 2014. Sources of the potential $\mathrm{CO}_{2}$ emission reduction in China: A nonparametric metafrontier approach. Applied Energy, 115(4): 491-501.

Hong L, Fang K N, Wei Y et al., 2013. Regional environmental efficiency evaluation in China: Analysis based on the Super-SBM model with undesirable outputs. Mathematical \& Computer Modelling, 58(5/6): 1018-1031.

IEA, 2012. World Energy Outlook 2012. Paris: International Energy Agency (IEA).

Ishii S, Tabushi S, Aramaki T et al., 2010. Impact of future urban form on the potential to reduce greenhouse gas emissions from residential, commercial and public buildings in Utsunomiya, Japan. Energy Policy, 38(9): 4888-4896.

Le G J, 2004. Space-time analysis of GDP disparities among European regions: A Markov chains approach. International Regional Science Review, 27(2): 138-163.

Li J, Zhou H, 2012. Correlation analysis of carbon emission intensity and industrial structure in China. China Population, Resources and Environment, 22(1): 7-14. (in Chinese)

$\mathrm{Li}$ T, 2013. Measuring the win-win performance of China's carbon reduction and economic growth with energy constraint: A range-adjusted measure of non-radial DEA. China Economic Quarterly, 12(2): 667-692. (in Chinese)

Liu Q Q, Wang S J, Zhang W Z et al., 2019. Examining the effects of income inequality on $\mathrm{CO}_{2}$ emissions: Evidence from non-spatial and spatial perspectives. Applied Energy, 236: 163-171.

Liu W B, Meng W, Li X X et al., 2010. DEA models with undesirable inputs and outputs. Annals of Operations Research, 173(1): 177-194.

Liu X, Li S M, 2015. An empirical analysis on the provincial energy efficiency in China based on SBM model of undesirable output. Mathematics in Practice and Theory, 45(2): 35-43. (in Chinese)

Liu Z, Geng Y, Xue B et al., 2011. A calculation method of $\mathrm{CO}_{2}$ emission from urban energy consumption. Resources Science, 33(7): 1325-1330. (in Chinese)

Meng M, Fu Y N, Wang T Y et al., 2017. Analysis of low-carbon economy efficiency of Chinese industrial sectors based on a RAM model with undesirable outputs. Sustainability, 9(3): 451.

Mielnik O, Goldemberg J, 1999. Communication the evolution of the "carbonization index" in developing countries. Energy Policy, 27(5): 307-308.

Ping W, Wu W S, Zhu B Z et al., 2013. Examining the impact factors of energy-related $\mathrm{CO}_{2}$ emissions using the STIRPAT model in Guangdong Province, China. Applied Energy, 106(11): 65-71. 
Ramanathan R, 2002. Combining indicators of energy consumption and $\mathrm{CO}_{2}$ emissions: A cross-country comparison. International Journal of Global Energy Issues, 17(3): 214-227.

Rey S J, 2010. Spatial empirics for economic growth and convergence. Geographical Analysis, 33(3): $195-214$.

Shen C, Feng R, Yu B B et al., 2018. Industrial $\mathrm{CO}_{2}$ emissions efficiency and its determinants in China: Analyzing differences across regions and industry sectors. Polish Journal of Environmental Studies, 27(3): 1239-1254.

Song J K, Liang L L, Niu D P et al., 2018. Evaluation, influencing factors and promotion countermeasures of cities' carbon emissions efficiencies in Shandong province. Journal of China University of Petroleum (Edition of Social Sciences), 34(1): 15-21. (in Chinese)

Stretesky P B, Lynch M J, 2009. A cross-national study of the association between per capita carbon dioxide emissions and exports to the United States. Social Science Research, 38(1): 239-250.

Su Y X, Chen XZ, Ye Y Y et al., 2013. The characteristics and mechanisms of carbon emissions from energy consumption in China using DMSP/OLS night light imageries. Acta Geographica Sinica, 68(11): 1513-1526. (in Chinese)

Sun J W, 2005. The decrease of CO emission intensity is decarbonization at national and global levels. Energy Policy, 33(8): 975-978.

Tone K, 2001. A slacks-based measure of efficiency in data envelopment analysis. European Journal of Operational Research, 130(3): 498-509.

Tone K, 2002. A slacks-based measure of super-efficiency in data envelopment analysis. European Journal of Operational Research, 143(1): 32-41.

Wang B, Lujin Y U, Yang Y S, 2013. Measuring and decomposing energy productivity of China's industries under carbon emission constraints. Journal of Financial Research, 10: 128.

Wang J Y, Wang S J, Li S J et al., 2019a. Evaluating the energy-environment efficiency and its determinants in Guangdong using a slack-based measure with environmental undesirable outputs and panel data model. Science of the Total Environment, 663: 878-888.

Wang Q W, Zhou P, Zhou D Q, 2010. Research on dynamic carbon dioxide emissions performance, regional disparity and affecting factors in China. China Industrial Economics, (1): 45-54. (in Chinese)

Wang Q W, Zhou D Q, Zhou P, 2011. Regional carbon dioxide emission performance and its reduction potential based on environmental production technology: The case of main industrial provinces in China. Studies in Science of Science, 29(6): 868-875. (in Chinese)

Wang S J, Fang C, Sun L et al., 2019b. Decarbonizing China's urban agglomerations. Annals of the American Association of Geographers, 109 (1): 266-285.

Wang S J, Huang Y Y, 2019. Spatial spillover effect and driving forces of carbon emission intensity at city level in China. Acta Geographica Sinica, 74(6): 1131-1148. (in Chinese)

Wang S J, Li Q Y, Fang C L et al., 2016. The relationship between economic growth, energy consumption, and $\mathrm{CO}_{2}$ emissions: Empirical evidence from China. Science of The Total Environment, 542: 360-371.

Wang S J, Liu X P, 2017. China's city-level energy-related $\mathrm{CO}_{2}$ emissions: Spatiotemporal patterns and driving forces. Applied Energy, 200: 204-214.

Wang S J, Liu X P, Zhou C S et al., 2017. Examining the impacts of socioeconomic factors, urban form, and transportation networks on $\mathrm{CO}_{2}$ emissions in China's megacities. Applied Energy, 185: 189-200.

Wang S J, Liu Y Y, Fang C L, 2015. Review of energy-related $\mathrm{CO}_{2}$ emission in response to climate change. Progress in Geography, 34(2): 151-164. (in Chinese)

Wang S J, Su Y X, Zhao Y B, 2018. Regional inequality, spatial spillover effects and influencing factors of China's city-level energy-related carbon emissions. Acta Geographica Sinica, 73(3): 414-428. (in Chinese)

Wang S J, Wang J Y, Fang C L et al., 2019c. Estimating the impacts of urban form on $\mathrm{CO}_{2}$ emission efficiency in the Pearl River Delta, China. Cities, 85: 117-129.

Wang Z F, Du Y Y, 2019a. Performance of carbon emission in Hunan province based on three-stage SBM model. Journal of Central South University of Forestry \& Technology (Social Sciences), 13(1): 23-30. (in Chinese)

Wang Z F, Du Y Y, 2019b. Spatial-temporal differences and influencing factors of carbon emission efficiency in 
Hunan province based on SBM-DEA model. Scientia Geographica Sinica, 39(5): 797-806. (in Chinese)

Wei X H, Yu L Z, 2011. Space dependence, carbon emissions and economic growth: Reinterpret China's EKC hypothesis. Probe, (1): 100-105.

Wei Y H D, Liao F H, 2012. Dynamics, space, and regional inequality in provincial China: A case study of Guangdong province. Applied Geography, 35(1/2): 71-83.

World B, 2010 . World Development Indicators 2010. Beijing: Chinese Financial \& Economic Publishing House.

Xu G Q, Liu Z Y, Jiang Z H, 2006. Decomposition model and empirical study of carbon emissions for China, 1995-2004. China Population, Resources and Environment, 16(6): 158-161. (in Chinese)

Xu S C, Xi R, He Z X, 2012. Influential factors and policy implications of carbon emissions for energy consumption in China. Resources Science, 34(1): 2-12. (in Chinese)

Yamaji K, Matsuhashi R, Nagata Y et al., 1993. A study on economic measures for $\mathrm{CO}_{2}$ reduction in Japan. Energy Policy, 21(2): 123-132.

Zeng G, Shang Y M, Si Y F, 2015. The convergent evolution of China's regional economic development models. Geographical Research, 34(11): 2005-2020. (in Chinese)

Zha J P, Tang F, 2012. Research on static level, dynamic change and affecting factors of industrial carbon emissions performance: An empirical analysis based on Chinese provincial panel data. Journal of Shanxi Finance and Economics University, (3): 71-80. (in Chinese)

Zhang J R, Zeng W H, Wang J N et al., 2017. Regional low-carbon economy efficiency in China: Analysis based on the Super-SBM model with $\mathrm{CO}_{2}$ emissions. Journal of Cleaner Production, 163: 202-211.

Zhang X L, 2012. Has transport infrastructure promoted regional economic growth? With an analysis of the spatial effects of transport infrasturcture. Social Sciences in China, (3): 60-77. (in Chinese)

Zhang Z Q, Qu J S, Zeng J J, 2008. A quantitative comparison and analysis on the assessment indicators of greenhouse gases emission. Journal of Geographical Sciences, 18(4): 387-399.

Zhao R Q, Huang X J, Peng B Z, 2012. Research on carbon cycle and carbon balance of Nanjing urban system. Acta Geographica Sinica, 67(6): 758-770. (in Chinese)

Zhao R Q, Huang X J, Zhong T Y, 2010. Research on carbon emission intensity and carbon footprint of different industrial spaces in China. Acta Geographica Sinica, 65(9): 1048-1057. (in Chinese)

Zhou C S, Shi C Y, Wang S J et al., 2018. Estimation of eco-efficiency and its influencing factors in Guangdong province based on Super-SBM and panel regression models. Ecological Indicators, 86: 67-80.

Zhou P, Ang B W, Han J Y, 2010. Total factor carbon emission performance: A Malmquist index analysis. Energy Economics, 32(1): 194-201. 